\title{
CONTRACTED SOCKET * + The Splint Method of Post-operative Control
}

B Y

F. MaXWell Lyons

DIRECTOR, MEMORIAL OPHTHALMIC LABORATORY, GIZA, CAIRO

ThE traditional Wheeler operation, an admirable concept upon which most later procedures have been based, was primarily intended for the obliterated socket in which no useful conjunctiva* remained and where complete relining of the reconstructed cavity with an epidermal graft was necessary. In the majority of distorted and contracted sockets resulting from civil and war injuries, however, a considerable amount of healthy conjunctiva persists. Whether this should be conserved or removed as a preliminary to grafting has long been a subject of controversy.

The widespread prejudice against mixing skin and mucous membrane on the grounds that it produces chronic irritation and discharge I have found to be largely unsubstantiated or, at least, over-stressed. On the other hand, the sacrifice of all existing conjunctiva has very definite disadvantages. Total relining, at its best, produces a capacious socket but one which lacks resilience and adaptability. The normal shrinkage of the graft adherent to the tarsal plates inevitably results in thickening of the lids and entropion which may be pronounced and, even in lesser degrees, detract from the final cosmetic effect.

In very few contracted sockets is there insufficient conjunctiva remaining to cover the tarsal plates, and for many years I have been convinced that this should be carefully preserved.

The greatest source of trouble in any form of socket reconstruction is post-operative shrinkage. As in all Thiersch grafting, about 30 per cent. shrinkage of the epidermal inlay must be accepted as " normal " and duly allowed for in planning the graft. Unless the bed is properly prepared and freed from scar tissue, this shrinkage will be much greater and little or no benefit may result from the operation.

In spite of all precautions and in the most favourable cases, post-operative contraction is seldom regular and, although the resultant socket may be adequate in size, its irregular shape will prevent the fitting of a standard artificial eye. The ordinary stent

* The acrylic moulds and the method of splint fixation described in this communication were demonstrated at the Meeting of the Egyptian Ophthalmological Society held in Cairo in March, 1946.

+ Received for publication, June 3, 1947. 
or acrylic mould with pressure bandage will not satisfactorily control this shrinkage and distortion. All of us must have witnessed at times the disheartening sight of rotation, tilting and even extrusion of the mould two or three weeks after an apparently successful operation.

The technique of grafting and post-operative splintage here described was designed to offer controlled resistance to the forces of contraction and to persuade the socket, from the start, to take

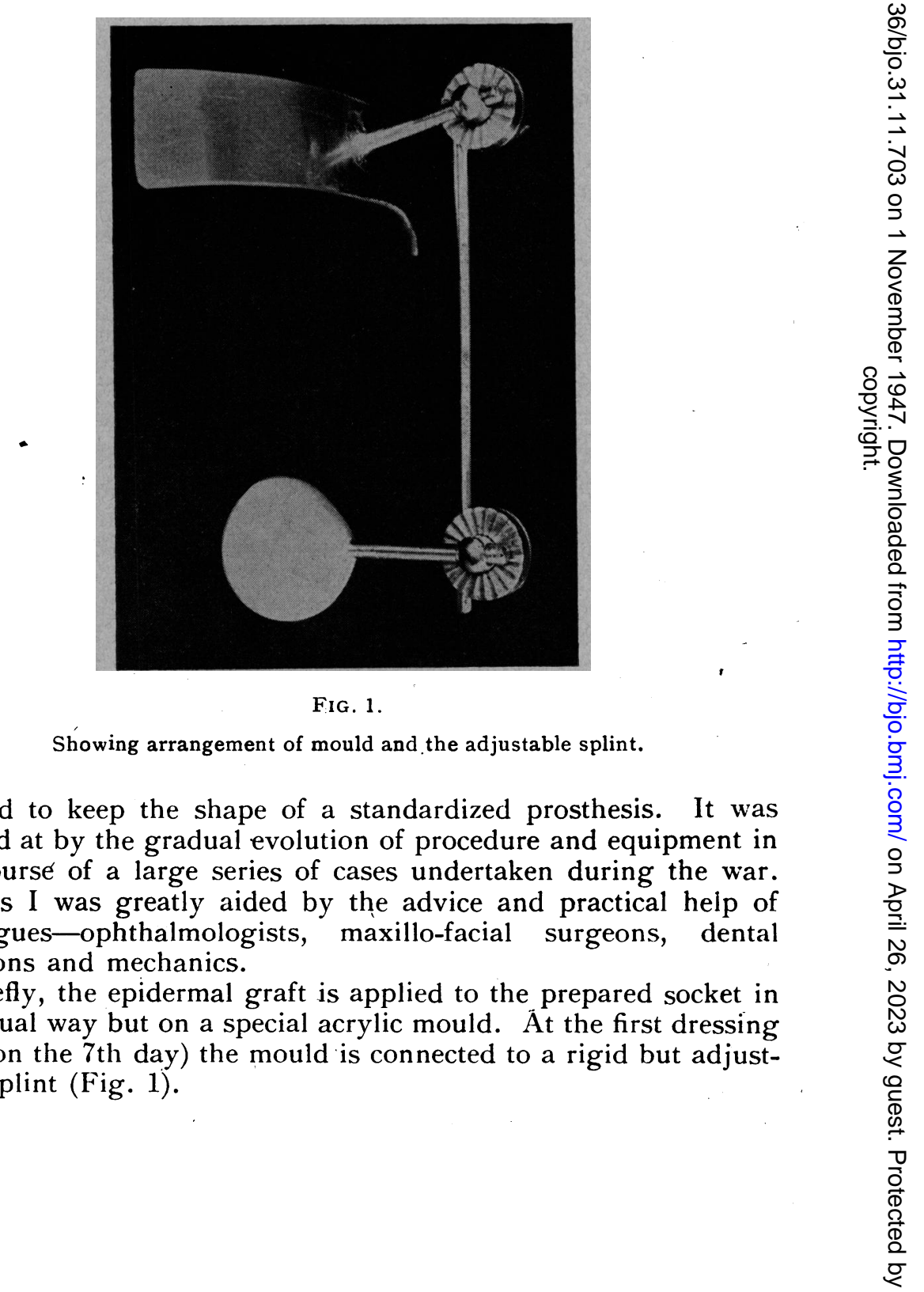

on and to keep the shape of a standardized prosthesis. It was arrived at by the gradual evolution of procedure and equipment in the course of a large series of cases undertaken during the war. In this I was greatly aided by the advice and practical help of colleagues-ophthalmologists, maxillo-facial surgeons, dental surgeons and mechanics.

Briefly, the epidermal graft is applied to the prepared socket in the usual way but on a special acrylic mould. At the first dressing (i.e., on the 7th day) the mould is connected to a rigid but adjustable splint (Fig. 1). 


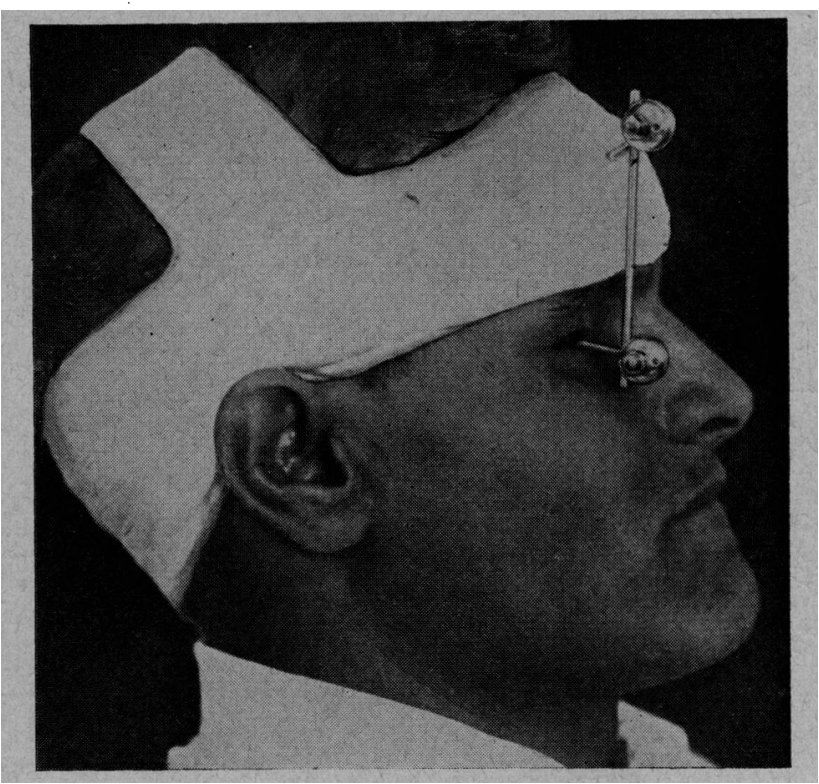

FIG. 2.

Plaster-of-paris head-cap and splint in position.

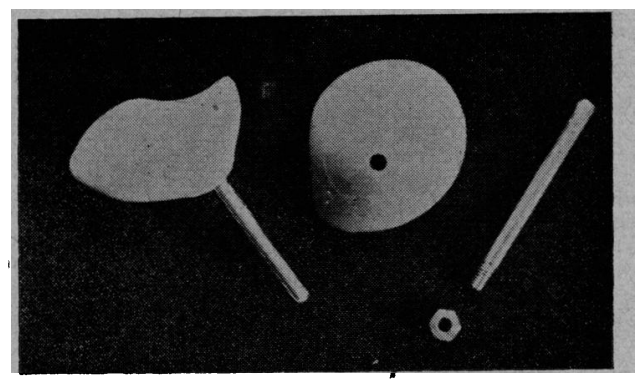

FIG. 3.

Showing details of mould and threaded rod. A nut similar to that shown in the photograph is embedded under the anterior surface of mould.

The fixed point is a frontal pin embedded in a plaster-of-paris head-cap and to this the mould is attached by stainless steel rods and universal clamps (Fig. 2).

The acrylic moulds are replicas of standard artificial eyes. Each mould has embedded below the anterior surface a small stainless steel nut (6 B.A.) into which a short length of rod, threaded at one end, can be screwed (Fig. 3). Latterly, Stallard's useful 
suggestion-of drilling holes through the thickness of the mould to allow seepage of exudate and discharge from the back of the socket-has been adopted although it is not shown in the figures. A stock of 12 moulds, 6 right, 6 left, of sizes varying from medium to large covers almost all requirements.

\section{Procedure}

Anaesthesia.-General anaesthesia is preferable throughout the operation. Local anaesthesia is less satisfactory owing to the difficulty of blocking the nerves of the donor area of the upper arm. As a compromise, which has often proved useful, the dissection and preparation of the socket may be carried out under local anaesthesia and intra-venous pentothal given for the final stages of cutting and placing the graft.

Operation.-A wide canthotomy is first performed. The conjunctiva is then incised along a line corresponding with the lower border of the lower tarsal plate, extending from the outer canthus up to and behind the caruncle. Through this incision dissection is carried down to the infra-orbital margin, the periosteum of which is exposed throughout its length. This is an important step since the adhesions formed here help to anchor the lower fornix. Any redundant tissue is excised so that the tarsal plate covered with its conjunctiva may assume its normal vertical position. The caruncle should be preserved if possible.

The upper part of the conjunctiva is then freed from adhesions over the muscle stump and scleral reminants, if any. It is undermined and the upper fornix reformed, leaving the conjunctiva to cover the upper tarsus. Dissection here should be backwards rather than directly upwards and care must be taken to avoid injury to the levator muscle.

The conjunctiva should be delicately handled and any traction applied by holding-sutures rather than by forceps since pieces of graft may become adherent to the abraded surface and they are difficult to remove later. All fibrous bands must be carefully removed.

The largest mould over which the lids will close and which fits snugly into the lower fornix is selected by trial. It is removed from the socket, fitted with its short threaded steel rod and placed on one side ready to receive the graft.

Sutures for closing the canthotomy are inserted but left untied. All oozing is checked and the socket is lightly insufflated with penicillin and sulphathiazole powder. The area is covered while the graft is prepared. 
A very thin epidermal graft, large enough to cover the mould and allow for overlapping, is cut. It is carefully eased off the knife into a bowl of warm saline so that it floats with its raw surface upwards. The mould, held by the attached rod, is insinuated underneath the graft and gently raised so as to lift the graft off the surface of the saline. With the aid of an iris repositor the graft can be evenly applied to the surface of the mould. If the graft is punctured over the posterior concavity of the mould it will readily subside into the slight hollow. Any redundant graft should be trimmed with scissors.

The socket is again examined to make sure that it is dry. Then, while an assistant retracts the lids, the graft and mould (still held by the rod) are lowered into the socket. The rod is unscrewed and removed, and the graft evenly spread over the anterior surface of the mould. The lids are closed and the canthotomy sutures are tied. It is not necessary to stitch the lids together although some prefer to do so. A tulle-gras and gamgee dressing is applied. Both eyes are covered with a firm crepe bandage.

The patient should be nursed flat or in a semi-recumbent position for the first two days; after that he may be allowed to sit up.

The dressings are left undisturbed until the 6th day. The bandages are then removed and the sound eye is uncovered. The dressing on the operated eye, if clean, is left in situ and fixed with adhesive strapping. A plaster-of-paris head-cap incorporating the metal frontal pin and plate is put on as shown in Fig. 2. First, a piece of stockinette is pulled over the head and over the ears. A single layer of crepe bandage is placed around the head and fixed with strapping; a similar piece of bandage is fixed across the top of the head in front of the ears. Next, a few layers of plaster-ofparis bandage are applied and reinforced over the frontal region. The pin and plate are now placed in position over the brow on the affected side and secured with several more layers of plaster bandage. For the sake of neatness, the stockinette is cut and folded back over the plaster and covered with a final layer or two of plaster bandage. It is left for 24 hours to harden. If the plaster is taken well down over the frontal region and under the occipital protuberance as shown in the figure, the head-cap will not lift off and will form a firm and steady support for the splint.

On the following day, i.e., the 7 th day after operation, the first dressing of the socket is done. The canthotomy sutures are removed. The lids are gently prised open and the rod is screwed into the hole in the mould. The mould is eased out of the socket. A pint of warm saline is poured over the socket and graft. Any pieces of redundant graft are removed. The excess saline is soaked up with a small piece of gauze. A few drops of penicillin (500 
$\mathrm{u} / \mathrm{cc}$.) are instilled. The mould (with the rod attached) is dipped into sterile liquid paraffin and reinserted in the socket. It is then connected to the frontal pin by means of a suitable length of rod and two universal clamps. The position of the mould is carefully adjusted and the clamps are tightened with a spanner.

From that moment treatment becomes easy. The patient is allowed up and about. No dressing is required except a couple of thicknesses of gauze. The socket is irrigated daily without removing the mould. 'Twice a week the splint is taken down and the mould removed and cleaned. The socket is washed out and the mould and splint replaced. Slight adjustments of pressure, etc., may be required day by day.

- There is no fixed time for keeping on the splint. Grafted sockets vary in their tendency to contract and in the period during. which contraction is likely to continue. After three weeks a test may be made :-The clamps are removed and the rod unscrewed from the mould. If, after an hour or two, the lids show signs of gaping, the splint should be reapplied for another week. If, on the other hand, there is no attempt at extrusion, the splint may be left off until the following day, but a careful watch must be kept. The need for further splinting is decided by the course of events. It is usually necessary to retain the splint in position for three to five weeks after operation. The plaster head-cap is not removed until all risk of contraction is past. An artificial eye may then be fitted.

The operative procedure I have described is applicable to the partially contracted socket in which there is sufficient conjunctiva remaining to line the lids. The technique of post-operative splinting, however, is equally suitable for the totally obliterated socket in which all conjunctiva is lost, but a word of warning should be given here. In such a case, the remnants of the tarsal plates must be grafted and some degree of contraction and entropion will inevitably follow. If the fornices are rigidly fixed by the splinted mould, the contraction will necessarily have its effect near the lid margin and the entropion may thus be increased. This should be looked for and if signs of it appear the pressure of the splint should be relaxed a little or, if necessary, the mould should be changed for one of slightly smaller size.

The equipment required for the splint is easy to obtain. Stainless steel rod in 12 inch lengths and the universal clamps are supplied by Messrs. Down Bros. The " 6 B.A." nuts may be obtained from most ironmongers. The moulds incorporating the nuts can be copied in acrylic resin from selected artificial eyes by a dental laboratory. The threaded rod to fit the moulds and the frontal plate and pin can easily be turned out by any jobbing mechanic from a length of steel rod and a small strip of sheet brass. 
The moulds and the rest of the splint can, of course, be used repeatedly.

\section{Summary}

1. The advisability of conserving healthy conjunctiva in a partially contracted socket is discussed.

2. Details of operation and a method of post-operative splinting of the mould are described.

\section{REFERENCES}

Wheeler, J. M.-Amer. Jl. Ophthal., Vol. IV, p. 481, 1921.

STALLARD, H. B.-" Eyè Surgery," p. 169, 1946.

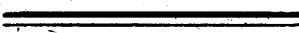

\section{ANNOTATION}

\section{The Milking-side Eye}

The milking-side eye of a cow is the right side. One not infrequently finds that people refer to sides of the body in terms connected with their daily work, The Admiral who complained of pain in a tooth on the port side and Commodore Trunnion, whose larboąrd eye was missing, are examples from the séafaring point of view. All those who were born and bred in country districts must have visited the cow house to see milking in progress during their childhood days. That, and visits to the forge, were among the diversions of our own youth. One remembers that the milker sat on a three legged stool on the right hand side of the animal with the cheek alongside the flank of the cow and face directed towards the caudal end of the beast. If milkers sat in any other position the cow would be unable to see what was going on, might get restive and upset the pail. The importance of this in these austerity days can hardly be overestimated.

A correspondent recently wrote to tell us of a farmer who had asked him to have a look at one of his heifers which had something wrong with its milking-side eye, and we heard later that the owner had developed a conjunctivitis in his own right eye. It may be recalled that Angel Clare chose the milking time to declare his affection for Tess. "Old Pretty by this time had looked round, puzzled; and seing two people crouching under her where, by immemorial custom, there should have been only one, lifted her hind leg crossly."

In view of the mechanical aids practised in milking at the present 